\title{
Versuche über die Dauer der postmortalen Erregbarkeit der Muskulatur verschiedener Fischarten bei Sauerstoffmangel.
}

\author{
Von \\ Dr. phil. et med. A. Willer. \\ (Aus dem tierphysiol. Institut der Landwirtsch. Hochschule, Berlin.)
}

(Mit 4 Textabbildungen.)

(Eingegangen am 1. August 1918.)

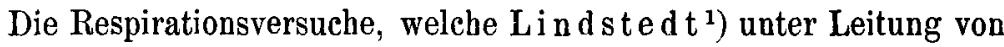
$\mathrm{Zuntz}$ und Cronheim an verschiedenen Fischarten ausgeführt hat, zeigten sehr erhebliche Unterschiede des Sauerstoffverbrauchs, sowohl bezogen auf gleiches Gewicht als auch auf gleiche Körperoberfläche. Auch die untere mit dem Leben noch verträgliche Grenze des Sauerstoffgehalts im Wasser war sehr verschieden, am niedrigsten bei den Cypriniden, am höchsten bei Forellen. Aber auch die ersteren gingen, im Gegensatz zu den Erfahrungen an Fröschen und vielen Wirbellosen bei Sauerstoffentziehung, zugrunde, ehe der Sauerstoffgehalt des Wassers ganz aufgebraucht war. Ein eigentlich anaërobes Leben ist also bei diesen Fischen nicht möglich, wahrscheinlich weil das Zentralnervensystem bei Sauerstoffmangel schnell seine Funktionen einstelit. Unter diesen Umständen erschien es wertvoll, das Verhalten der Muskulatur von Fischen mit verschieden starkem Stoffwechsel bei Sauerstoffmangel zu erforschen. Die Versuchsanordnung war folgende: In dem stets gleichen Raume und unter mit einer Ausnahme (statt $18-20^{\circ}$ $21-23^{\circ}$ C.) stets gleichen Temperaturverhältnissen wurden die einzelnen Versuchsfische mit dem Induktionsstrom gereizt. Zur Reizung wurde das Du Bois-Reymond'sche Schlitteninduktorium

1) $\mathrm{Ph}$. Lindstedt, Untersuchungen über Respiration und Stoffwechsel von Kaltblütern. Zeitschr. f. Fischerei 14. Jahrg. H. 3. 1913/14. 
benutzt, und zwar wurden stets tetanisierende Reize verwendet. Anfang und Ende einer jeden Reizung wurde durch einen Vorreiberschlüssel von Du Bois-Reymond bewirkt. Das Anlegen der beiden als Elektroden dienenden Kupferdrähte wurde stets unter möglichst gleichen Bedingungen und an gleicher Stelle, soweit dies möglich war, vorgenommen, und zwar wurde der eine Draht durch ein Loch gezogen, das durch die Dorsalmuskulatur vor einer auf dem Ansatz der Brustflossen senkrechtstehenden Linie gebohrt wurde, der andere Draht möglichst dicht an der Schwanzflosse ventral von der Wirbelsäule durch die Muskulatur. Auf diese Weise wurde versucht, unter möglichst gleichen Verbältnissen zu arbeiten.

Die Länge der Fische wurde annähernd gleich genommen, abgesehen von den Versuchen mit Aal und Hecht. Der Sauerstoffabschluss wurde dadurch erreicht, dass der geköpfte und des Herzens beraubte Versuchsfisch in einer Glasschale mit Paraffinum liquidum übergossen wurde. Um etwa im Blut noch vorhandenen Sauerstoff $z \mathfrak{u}$ entfernen, wurde vor Beginn des eigentlichen Versuches der ganze Fisch nach der Dekapitation kurze Zeit tetanisiert. War dies geschehen, so wurden dem Versuchsfisch die zur Anbringung der Elektroden nötigen Löcher durch die Muskulatur gebohrt, die Elektroden angebracht und kurze Zeit tetanisiert.

Die Prüfung der Reizschwelle wurde stets eine Viertelstunde nach Tötung des Fisches begonnen, so dass die erste Ablesung, wenn der Fisch um 10 Uhr getötet war, um 10 Uhr 15 Minuten vorgenommen wurde. Es wurde hierbei einmal bestimmt, bei welchem äussersten Abstand der sekundären Spirale von der primären noch gerade Bewegung zu beobachten war. Also wenn zum Beispiel bei $22,5 \mathrm{~cm}$ Rollenabstand noch eine kleinste Bewegung der Schwanzspitze erkennbar war, bei $22,6 \mathrm{~cm}$ nicht mebr, so wurde notiert: "Minimalreaktion $=22,5 \mathrm{~cm}$ ". Abgelesen wurde diese "Minimalreaktion" an derjenigen Flossenspitze - die Flossenmuskulatur reagierte stets am längsten -, die am empfindlichsten reagierte. Es war dies in vielen Fällen die Schwanzflosse, jedoch nicht bei allen untersuchten Arten.

Beim Karpfen fand sich die interessante Tatsache, dass zuerst die untere Schwanzspitze die Reaktion bei weitestem Rollenabstand gab; nach einiger Zeit jedoch war es nicht mehr die Schwanzflosse, sondern die Rückenflosse, die diese Minimalreaktion zeigte, bis dann gegen das Ende hin wieder die Schwanzflosse diese ergab, um jedoch mit 
der Rückenflosse zugleich ihre Bewegung bei Reizung endgültig einzustellen. Auf die näheren Einzelheiten wird noch bei Besprechung der Kurven einzugeben sein.

Neben der Notierung der "Minimalreaktion" wurde auch ermittelt, bei welchem Rollenabstand noch eine Reaktion aller Körpermuskeln in ihrer Gesamtheit eintrat. Hierzu ist zu bemerken, dass bei dieser Ablesung die subjektive Anschauung des Beobachters eine grosse Rolle spielt, da es mitunter schwer zu sagen ist, ob noch die Gesamtmuskulatur reagiert oder nicht. Da jedoch stets von dem gleichen Beobachter abgelesen wurde, so ist vielleicht diese Fehlerquelle für den Vergleich der erhaltenen Kurven möglichst eingeschränkt worden, da ich mir Mühe gab, die Frage, ob noch die gesamte Muskulatur reagiere oder nicht, nach gleichen Gesichtspunkten zu entscheiden.

Die beiden Ablesungen, die der "Minimalreaktion" und die der Reaktion der gesamten Körpermuskulatur, wurden alle 15 Minuten vorgenommen. Die erhaltenen Notierungen wurden auf Millimeterpapier aufgetragen, und zwar bedeuten hier die halben Millimeter der Ordinate den Rollenabstand in Zentimetern, die der Abszisse die Zeit, indem je 5 Millimeter gleich einer Viertelstunde gerechnet wurden. Die entsprechend den einzelnen Messungen eingetragenen Punkte wurden durch Linien miteinander verbunden, und es wurden auf diese Weise Kurven gewonnen, welche einen Vergleich untereinander gestatteten.

Bei einzelnen Versuchen wurden in Zwischenräumen von je 30 Minuten die betreffenden Fische durch andauernden Tetanus so lange gereizt, bis eine völlige Ermüdung der Muskulatur eintrat. Diese Reizung wurde einerseits vorgenommen, um zu studieren, nach welcher Zeit eine Erholung und inwieweit sie eintrat, andererseits, um zu ersehen, welchen Einfluss diese Ermüdungsversuche auf die Dauer der Reizbarkeit der Muskulatur und die Form der schliesslich erlangten Kurve hätten. Hierauf wird späterhin noch näher einzugehen sein.

Der Nullpunkt der Ordinaten meiner Kurven entspricht der Nullmarke des Schlitteninduktoriums, d. h. dem vollkommenen Überschieben der sekundären Spirale über die primäre. Das Ausbleiben der Reaktion in dieser Stellung wurde durch einen Punkt $5 \mathrm{~mm}$ unter der Abszisse bezeichnet, während der Punkt auf die Abszisse gelegt wurde, wenn roch eine Reaktion bei übereinandergeschobenen Rollen eintrat. 
Versuche über die Dauer der postmortalen Erregbarkeit der Muskulatur usw. 403

Der Schluss der Kurve wird aber auch noch dadurch weniger genau, dass man bei viertelstündlicher Ablesung nicht sagen kann, in welcher Minute dieses viertelstündlichen Zeitraumes die Reizbarkeit aufgehört hat. Es ist also wiederum eine gewisse Eigenmächtigkeit, den Zeitpunkt des Erlöschens so einzutragen, als ob er eine Viertelstunde nach dem letzten beobachteten Ausschlag eingetreten wäre. Ich bin jedoch der Ansicht, dass diese Ungenauigkeit bei der Behandlung der vorliegenden Frage: Wie verbält sich die Körpermuskulatur einzelner Fische unter anoxybiotischen Verhältnissen? ziemlich gleichgültig ist.

Der Vollständigkeit halber wäre noch anzugeben, dass die Versuchsfische sämtlich vor den anzustellenden Reizversuchen einige (4-5) Tage gehungert hatten und unter gleichen Verhältnissen aufbewahrt waren, so dass ihr Zustand im allgemeinen, soweit dies zu erreichen ist, ungefähr der gleiche gewesen ist ${ }^{1}$ ). Angestellt wurden die Versuche in der Zeit von Ende Januar bis Anfang Mai.

Von den einzelnen Fischarten wurden versehiedentlich mehrere Exemplare gepruft, um zu erkennen, ob etwa grössere individuelle Unterschiede vorhanden seien. Es hat sich nun hierbei gezeigt, dass die Form der erlangten Kurven bei den einzelnen Vertretern jeder Art im allgemeinen die gleiche, und dass auch die Zeit, während der die Muskulatur für den Induktionsstrom reaktionsfähig bleibt, bis auf kleine Unterschiede im allgemeinen gleich lang ist. Dies gilt sowohl für die "Minimalreaktion" als auch für die Reaktion der gesamten Körpermuskulatur. Einen Einfluss auf die Zeitdauer hat es jedoch, wenn $\mathrm{zu}$ den Versuchen nicht frische, gesunde Fische genommen werden, sondern wenn man solche auswählt, die durch langen und schlechten Transport sehr geschwächt sind, oder die zum Beispiel stark von Saprolegnien befallen sind. Der Charakter der Kurve jedoch bleibt ein ähnlicher auch bei den geschwächten oder kranken Exemplaren; nur sind die Abstürze stärker ausgeprägt und die sanfter verlaufenden Teile infolgedessen stärker betont und schärfer abgesetzt. - Der Wechsel zwischen diesen stärkeren und schwächeren Neigungen in der Kurve bleibt demnach in der Reihenfolge derselbe und ist an geschwächten Exemplaren besonders gut zu erkennen.

Es wurde schon oben erwähnt, dass halbstündlich bei einigen

1) Ausnahmen sind jeweilig angegeben.

Pflüger's Archiv für Physiologie. Bd. 173. 
Versuchsfischen eine bis zur vollständigen Ermüdung der Muskulatur gehende Tetanisierung vorgenommen wurde. Es ist nun interessant, zu sehen, dass diese balbstündliche Erschöpfungstetanisierung sowohl auf den Charakter der Kurve als auch auf die Zeitdauer der Reaktionsfähigkeit der Muskulatur keinen oder doch wenigstens keinen wesentlichen Einfluss hat. Der einzige und in den meisten Fällen deutlich erkennbare Einfluss ist der, dass zu derselben Zeit, wo in der Kurve eines Versuchsfisches von der gleichen Art, bei dem diese Erschöpfungstetanisierung nicht gemacht wurde, ein charakteristischer, stärkerer Abfall erfolgt, dieser ein wenig ausgeprägter und intensiver ist. Ich verweise in dieser Beziehung im übrigen auf die folgende Einzelbesprechung der Versuche.

\section{Tinca tinca, der Schlei.}

Zur Untersuchung kamen zwei Schleie, der eine Ende Januar, der andere Anfang März. Da der Reizversuch im Januar überhaupt erst den Anstoss zu vorliegenden Untersuchungen gab, so leidet er noch an einigen Unvollkommenheiten, die bei den späteren Versuchen fortfallen konnten. So musste diese erste Versuchsreihe wegen anderweitiger Inanspruchnahme vor dem völligen Aufhören und Verschwinden der Minimalreaktion abgebrochen werden. Auch waren nicht sofort die Zahlen, die den Rollenabstand bei minimalster Reaktion angeben, notiert worden. Infolgedessen fehlt der erste Teil der Kurve wie der Schlussteil; sie beginnt erst $\mathbf{1}^{\mathbf{1}} / \mathbf{4}$ Stunden nach der Tötung des Fisches. Ich verzichte deshalb auf Wiedergabe der Kurve und verweise auf Tab. 1. Der erbaltene Teil der Beobachtungsreihe jedoch konnte zum Vergleich mitherangezogen werden. Wird jedoch von den angegebenen Unvollkommenheiten von Versuch 1 abgesehen, so ist doch noch ein für unsere Untersuchungen beabsichtigter Unterschied zwischen den beiden an Schleien vorgenommenen Reizversuchen vorhanden. Bei dem ersten Versuch nämlich wurde die schon oben besprochene Ermüdungstetanisierung vorgenommen. Ein Kreuz am oberen Rand der Kurvenfigur bezeichnet den jedesmaligen Ermüdungstetanus. Die äusseren Bedingungen waren bei beiden Schleiversuchen die gleichen: Die Temperatur im Raum beim ersten betrug $18-20^{\circ} \mathrm{C}$., beim zweiten $19-20^{\circ} \mathrm{C}$; die Länge des ersten Fisches war $25 \mathrm{~cm}$, die des zweiten $25,5 \mathrm{~cm}$. Nur war der erste Schlei längere Zeit im Aquarium gehalten worden als der zweite. Diese das Wohlbefinden 
Versuche über die Dauer der postmortalen Erregbarkeit der Muskulatur usw. 405

des Fisches sicher beeinträchtigenden ungünstigen Verbältnisse. in der Gefangenschaft, denen dieser länger ausgesetzt war als der zweite Schlei, haben, wie ich wohl glaube annehmen zu dürfen, den niedrigeren Wert im Rollenabstand veranlasst, denn bei Beginn der Kurve I, also 11/4 Stunden nach der Tötung des Fisches, ist der Rollenabstand $21,5 \mathrm{~cm}$, während er zu dieser Zeit bei dem zweiten Schlei $28,9 \mathrm{~cm}$ beträgt; der Unterschied zwischen den Rollenabständen beträgt also $7,4 \mathrm{~cm}$.

Wie sich aus den späteren Kurven ergibt (s. Kurven von Leuciscus rutilus - hier beträgt die Differenz im Rollenabstand anfangs $8,3 \mathrm{~cm}$ ), ist der Anfang der Kurve stets niedriger, wenn die Fische in der Gefangenschaft längere Zeit gehalten wurden, als wenn sie frisch zu den Versuchen benutzt werden konnten. Auch ist biervon die Zeitdauer der Reizbarkeit bis zu gewissen Grenzen abhängig, während das Charakteristische an der Kurvenform dauurch wenig oder gar nicht verändert wird, höchstens, dass ähnlich wie durch den halbstündigen Ermüdungstetanus die charakteristischen Absturze ausgeprägter werden. Diese Absätze in den Kurven nun werden bei den durch die längere Gefangenschaft und Hunger geschwächten Fischen, zum Teil wobl infolge der Verkürzung in der Zeitdauer, um ein Geringes nach dem Anfang hin verschoben, wie sich auch späterhin noch deutlicher zeigen wird. In Tab. 1 sehen wir 1 Uhr 15 Minuten, das heisst $\mathbf{1}^{3} / 4$ Stunden nach der Köpfung ein Sinken der Erregbarkeit, die 1 Uhr 30 Minuten fast wieder den früheren Wert erreicht hat. Dieses Verhalten beruht auf einer ausserhalb des Versuches liegenden besonderen Inanspruchnahme durch Reizung.

Die Ergebnisse des anderen Schleiversuchs stellen die zwei Kurven Abb. 1 dar.

Diese sind vollständig vom Anfang des Versuches bis zum Schluss durchgefübrt. Wir sehen, dass die Kurve der Minimalreaktion in ihrem Verlauf nicht regelmässig und in gleichem Maasse abfallend ist, sondern, dass ihr im allgemeinen sanftes Absteigen zur Nullinie durch einen plötzlichen, stärkeren Abfall, der am Schluss sogar als Absturz bezeichnet werden kann, unterbrochen wird. Schon im Beginn der Kurve ist im Vergleich zum allgemeinen Verlauf ein solcher Abfall, der jedoch nur kurze Zeit anhält, zu beobachten. Darauf erfolgt ein längeres, ziemlich gleichmässiges Absinken, das $4^{1 / 2}$ Stunden anhält, um dann für eine Zeit von $3 / 4$ Stunde stärker zu werden. Nach dieser Zeit wird das 
Fallen der Kurve wiederum sanfter, um allmählich jedoch steiler und steiler zu werden, bis ziemlich plötzlich 10 Stuncien nach Beginn des Versuches ein Absturz erfolgt, der nach 30 Minuten zum völligen Erlöschen der Reaktionsfähigkeit führt. Die Kurve zeigt also, dass ihr im allgemeinen gleichmässiger Verlauf dreimal gestört wird durch ein stärkeres Absinken, und zwar gleich zu Beginn, ungefähr in der Mitte der Versuchszeit und am Schluss. Diese drei Abstürze sind deswegen besonders interessant, weil sie sich mehr oder minder in gleicher Weise bei den übrigen Cyprinidenkurven vorfinden. Inwieweit diese Erscheinung auch bei Vertretern anderer Familien auftritt und zum Vergleich heranzuziehen ist, werden wir späterhin noch besprechen.

1. Versuch. 24. Januar 1914. Temperatur $18-20^{\circ} \mathrm{C}$. Tötung des Fisches 11 Uhr 30 Minuten vormittags.

Aus Tab. 1 geht hervor, dass eine Reaktion der gesamten Muskulatur auf die Reizung mit dem Induktionsstrom hin festzustellen ist während einer Zeit von $4^{3 / 4}$ Stunden (von 11 Uhr 30 Minuten bis 4 Uhr 15 Minuten). Die zur Erzielung dieses Effekts nötige Stromstärke wächst langsam in den ersten 3 Stunden nach dem Tode, viel stärker und immer mehr, zunehmend bis zum Erlöschen dieser Reaktion um 4 Uhr 30 Minuten.

Es ist nun noch auf die Ermüdungsversuche einzugehen, die schon oben erwähnt worden sind. Da zeigt sich, dass zu einer völligen Erschöpfung, d. h. bis absolut keine Reaktion mehr zu erkennen ist, zuerst 40 , ja 70 Sekunden währende Dauerreizung (Tetanisierung) erforderlich ist, dass aber je längere Zeit seit der Tötung des Fisches verflossen ist, eine um so kürzere Reizzeit zur Erlangung einer vollständigen Ermüdung notwendig ist. Merkwürdig ist dabei jedoch, dass dem die Länge der Zeit, die zur Erholung, d. h. zur Wiedererlangung der der Versuchszeit entsprechenden Reaktionsfähigkeit der Muskulatur, notwendig ist, nicht entspricht. Im Gegenteil, das Verhältnis der Reizzeit, die zur völligen Ermüdung notwendig ist, zur Ruhezeit, die zur Wiedererholung gebraucht wird, wird immer kleiner. Es steht also die Erholungszeit zur Ermüdungszeit in keinem konstanten Verhältnis. Doch scheint die erstere abhängig zu sein von der Ermüdungszeit insofern, als sie nämlich abnimmt bei kürzere Zeit dauernder Reizung. Ich hatte eigentlich erwartet, dass mit der Länge der Zeit, die seit der Tötung verflossen ist, die Erholungszeit zunehmen müsste. 
Versuche über die Dauer der postmortalen Erregbarkeit der Muskulatur usw. 407

Tabelle 1 .

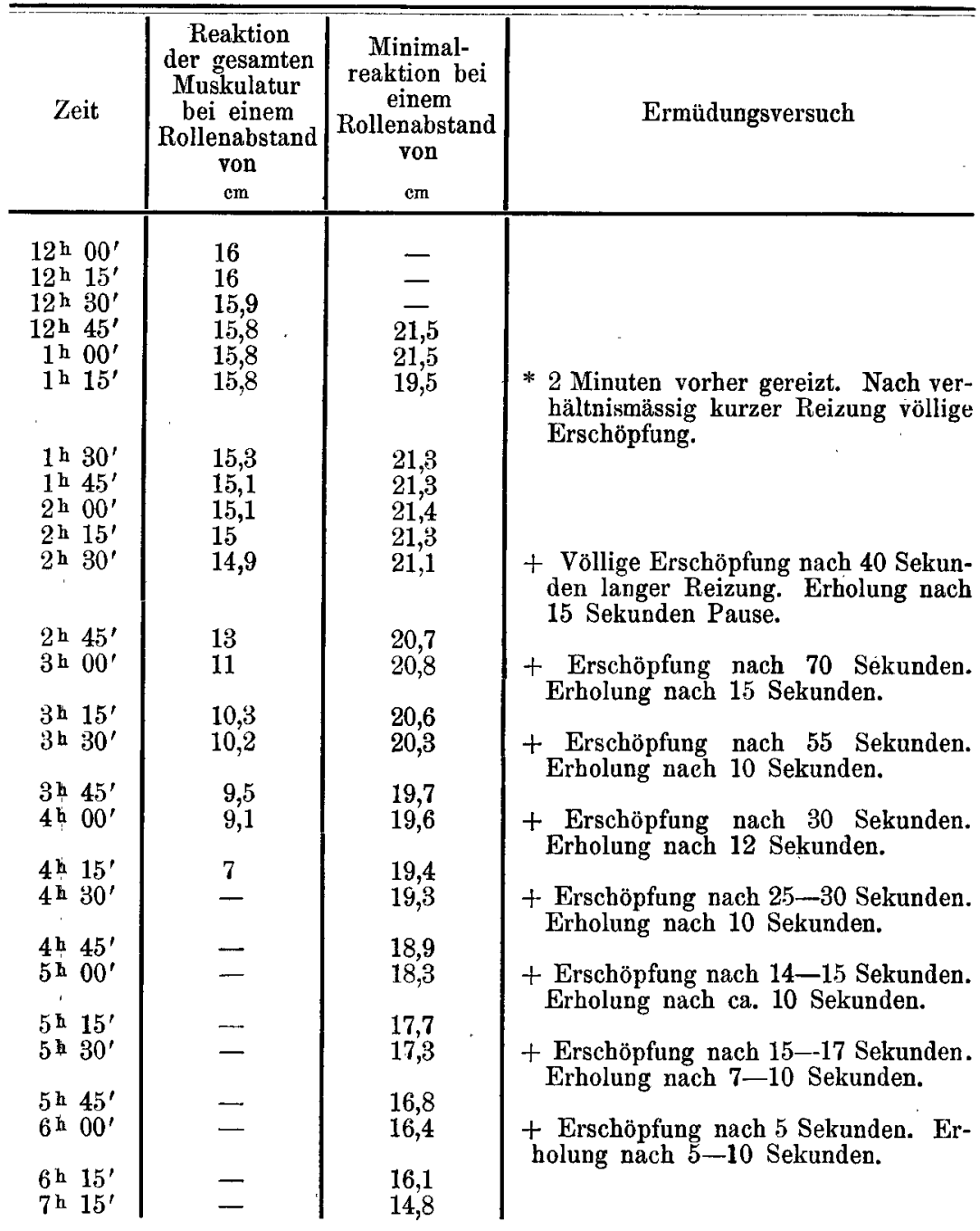

Der Ermüdungsversuch wurde stets, auch in den noch später zu schildernden Fällen, so ausgeführt, dass bei der Reizung die sekundäre Rolle des Induktionsapparates ganz über die primäre geschoben wurde, dass also mit dem stärksten Strom gereizt wurde. Vorgenommen wurde er stets nach der zur Herstellung der Kurven dienenden Hauptablesung.

Die Notierungen des zweiten an einem Schlei ausgeführten Versuches (9. März 1914) sind aus Abb. 1 (S. 408) zu entnehmen. 
Bei diesem zweiten Versuch reagierte also die gesamte Muskulatur auf die Reizung mit dem Induktionsstrom während einer Zeit von $3^{1 / 4}$ Stunden (von 8 Uhr 5 Minuten bis 11 Uhr 20 Minuten), während die "Minimalreaktion" erst nach $10^{1 / 2}$ Stunden erlosehen war. Wie schon oben gesagt wurde, sind die Fehlerquellen bei der Ablesung der Reaktion der gesamten Muskulatur notwendigerweise gross, und ich möchte daher keinen allzu grossen Wert auf die Einzelheiten dieser Kurven legen.

\section{Leuciseus rutilus, die Plötze.}

Zur Untersuchung der Verhältnisse bei der Plötze wurden Versuche mit zwei Exemplaren vorgenommen, der eine Versuch am 6. März, der andere am 13. März 1914. Beide sind vollständig

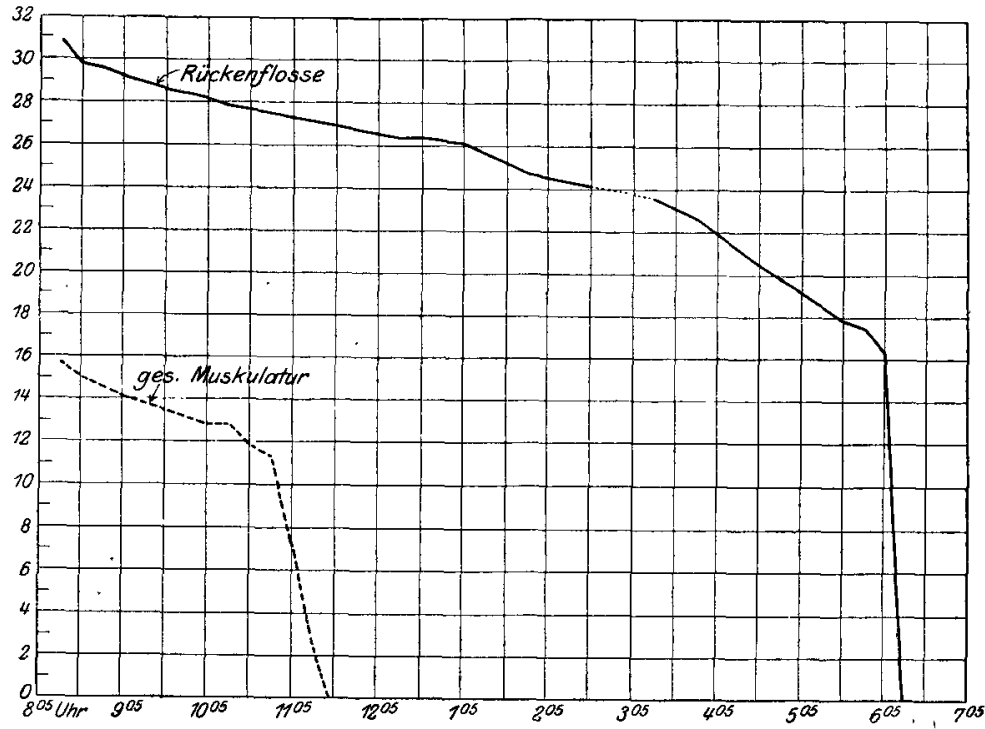

Abb. 1. Versuch vom 9. März 1914.

durcbgeführt. Die erste Plötze, mit der am 6. März gearbeitet wurde, war etwas länger als die übrigen Fische im Aquarium gehalten worden und dadurch vielleicht ein wenig geschwächt. Bemerkenswert ist, dass zu Anfang der Versuche bei der ersten Reizung der Fisch mit einer blitzartigen Bewegung kräftig den ganzen Körper hochschnellte, was ausser bei der Plötze Nr. 2 nur noch bei der Bachforelle und der einen Regenbogenforelle beobachtet werden konnte. Bei allen 
Versuche über die Dauer der postmortalen Erregbarkeit der Muskulatur usw. 409

übrigen Versuchsfischen trat auch zu Beginn nur eine mehr oder weniger kräftige Kontraktion der Muskulatur ein, ohne dass es zu einer derartig koordinierten Körperbewegung kam. Diese Allgemeinbewegung des Fischkörpers dürfte wohl darauf beruhen, dass das Rückenmark noch reizbar war.

Die Temperatur des Versuchsraumes betrug bei der ersten Plötze $20^{\circ} \mathrm{C}$. bei der zweiten $19-20^{\circ} \mathrm{C}$. Die Länge der ersten war $29 \mathrm{~cm}$, die der zweiten $24,5 \mathrm{~cm}$. Es sollen nun zuerst die Notierungen des ersten Plötzenversuches folgen:

Versuch am 6. März 1914. Temperatur $20^{\circ}$ C. Tötung des Fisches 11 Uhr 55 Minuten vormittags.

Tabelle 2.

\begin{tabular}{|c|c|c|c|c|c|}
\hline Zeit & $\begin{array}{c}\text { Reaktion } \\
\text { der gesamten } \\
\text { Muskulatur } \\
\text { bei einem } \\
\text { Rollenabstand } \\
\text { von } \\
\text { cm }\end{array}$ & $\begin{array}{c}\text { Minimal- } \\
\text { reaktion bei } \\
\text { einem } \\
\text { Rollenabstand } \\
\text { von } \\
\text { cm }\end{array}$ & $\begin{array}{l}\text { Ermüdungs- } \\
\text { versuche }\end{array}$ & Zeit & $\begin{array}{l}\text { Minimal- } \\
\text { reaktion bei } \\
\text { einem } \\
\text { Rollenabstand } \\
\text { von } \\
\text { cm }\end{array}$ \\
\hline $\begin{array}{ll}12^{\mathrm{h}} & 10^{\prime} \\
12^{\mathrm{h}} & 25^{\prime}\end{array}$ & $\begin{array}{l}15 \\
11,1\end{array}$ & $\begin{array}{l}21,2 \\
21\end{array}$ & $\begin{array}{c}\text { + Völlige Er- } \\
\text { schöpfung nach } \\
90 \text { Sekunden. } \\
\text { Erholung nach } \\
20 \text { Sekunden. } \\
\text {. }\end{array}$ & $\begin{array}{l}3 \text { h } 25^{\prime} \\
3 \text { h } 40^{\prime}\end{array}$ & $\begin{array}{l}6,6 \\
5\end{array}$ \\
\hline $\begin{aligned} 12 \mathrm{~h} & 40^{\prime} \\
12 \mathrm{~h} & 55^{\prime} \\
1 \mathrm{~h} & 10^{\prime} \\
1 \mathrm{~h} & 25^{\prime} \\
1 \mathrm{~h} & 40^{\prime} \\
1 \mathrm{~h} & 55^{\prime} \\
2^{\mathrm{h}} & 10^{\prime} \\
2 \mathrm{~h} & 25^{\prime} \\
2^{\mathrm{h}} & 40^{\prime} \\
2^{\mathrm{h}} & 55^{\prime} \\
3 \mathrm{~h} & 10^{\prime}\end{aligned}$ & $0-$ & $\begin{array}{l}15,2 \\
15,3 \\
15 \\
14,4 \\
14,3 \\
12,1 \\
12,1 \\
12 \\
10,3 \\
8,2 \\
7,6\end{array}$ & & $\begin{array}{l}3 \mathrm{~h} 55^{\prime} \\
4^{\mathrm{h}} 10^{\prime} \\
4^{\mathrm{h}} 25^{\prime} \\
= \\
= \\
= \\
= \\
= \\
=\end{array}$ & $\begin{array}{l}3 \\
2 \\
0- \\
- \\
- \\
- \\
= \\
= \\
= \\
-\end{array}$ \\
\hline
\end{tabular}

Eine Reaktion der gesamten Muskulatur auf die Reizung mit dem Induktionsstrom ist also nachweisbar während einer Zeit von $1 / 2$ Stunde (von 11 Uhr 55 Minuten bis 12 Uhr 25 Minuten). Die „Minimalreaktion" erlosch erst nach $4^{1 / 4}$ Stunden. Bei dieser Plötze wurde ein Ermüdungsversuch gemacht und sollte eigentlich wie bei dem ersten Schlei weiter fortgeführt werden. Es zeigte sich jedoch nach der ersten Ermudungsreizung, dass die Reaktion sehr schlecht geworden war, so dass auf eine Weiterfuhrung verzichtet wurde. Geschwächte 
Individuen scheinen derartige Ermüdungsversuche sehr schlecht zu vertragen. Allerdings musste zur vollen Erschöpfung der Reaktionsfähigkeit 1 Minute 30 Sekunden lang gereizt werden, wonach jedoch keine vollständige Erholung eintrat, sondern nur eine geringe nach 20 Sekunden. Die Reizbarkeit zeigt auch hier einen ganz besonders starken Abfall. Der anfängliche Rollenabstand fự die Minimalreaktion liegt bei $21,2 \mathrm{~cm}$ gegen 29,5 am 13 . März, was ebenfalls, wie schon oben besprochen worden ist, auf die Schwächung des Fisches durch die längere Gefangenschaft zurückzuführen ist. Auch hier findet sich dreimal ein schneller Abfall der Reizbarkeit, ähnlich dem beim Schlei Nr. 2 beobachteten. Der erste ist sofort zu Beginn des Versuches gelegen,

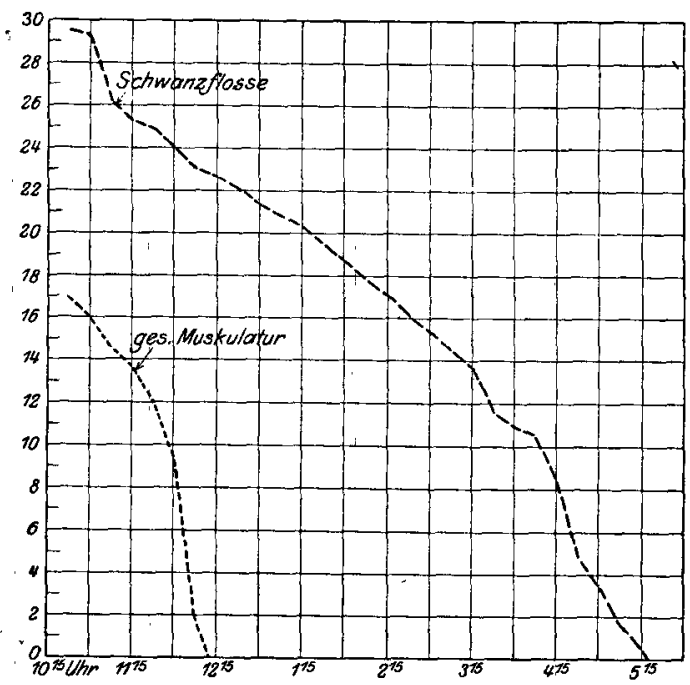

Abb. 2. Versuch am 13. März 1914.

der zweite ungefähr in der Mitte der Versuchszeit, der dritte gegen das Ende hin. Während jedoch beim Schlei der dritte starke Kurvenabfall die Kurve beschliesst, ist dies bei der Plötze nicht der Fall, sondern es folgt schliesslich noch ein sanfteres Absteigen, s. auch Abb. 2, welche über den zweiten Versuch mit Leuciscus Aufschluss gibt.

Die gesamte Muskulatur hatte also ihre Reaktionsfähigkeit nach $1^{3 / 4}$ Stunden verloren; die letzte Reaktion überhaupt trat nach 7 Stunden auf. Über den Verlauf der Kurve ist nichts hinzuzufügen; die allgemeine Form ist die gleiche wie bei der ersten Plötze. 
Versuche über die Dauer der postmortalen Erregbarkeit der Muskulatur usw. 411

\section{Cyprinus carpio, der Karpfen.}

Vom Karpfen wurden gleichfalls zwei Exemplare untersucht; die Länge des einen betrug $27 \mathrm{~cm}$, die des anderen $24 \mathrm{~cm}$. Die Temperatur des Versuchsraumes war am 3. April $18-20^{\circ}$, am 20. April 21-23 ${ }^{\circ}$. Wie ersichtlich, hat jedoch dieser kleine Temperaturunterschied keinen Einfluss auf die Dauer der Reaktionsfähigkeit wie auf die Form der Kurven gehabt. Es folgen die Notierungen über den ersten Karpfen:

Versuch am 3. April 1914. Temperatur $18-20^{\circ} \mathrm{C}$. Tötung des Fisches 9 Uhr 50 Minuten vormittags.

Tabelle 3 .

\begin{tabular}{|c|c|c|c|c|c|c|}
\hline Z eit & $\begin{array}{l}\text { Reaktion } \\
\text { d. gesamt. } \\
\text { Musku- } \\
\text { latur bei } \\
\text { pinem } \\
\text { Rollen- } \\
\text { abstand } \\
\text { von } \\
\text { cm }\end{array}$ & $\begin{array}{l}\text { Minimal- } \\
\text { reaktion } \\
\text { bei einem } \\
\text { Rollen- } \\
\text { abstand } \\
\text { ron } \\
\mathrm{cm}\end{array}$ & $\begin{array}{l}\text { Ermüdungs- } \\
\text { versuche }\end{array}$ & Zeit & $\begin{array}{c}\text { Minimal- } \\
\text { reaktion } \\
\text { bei einem } \\
\text { Rollen- } \\
\text { abstand } \\
\text { von } \\
\text { cm }\end{array}$ & $\begin{array}{c}\text { Ermüdungs- } \\
\text { versüche }\end{array}$ \\
\hline $10^{\text {h }} 05^{\prime}$ & 15,8 & 24,1 & & $1 \mathrm{~h} 35^{\prime}$ & 12,4 & $\begin{array}{l}\text { Vollständige Er. } \\
\text { müdung nach } 5 \text { Se- } \\
\text { kunden. Erholung } \\
\text { fast momentarl. }\end{array}$ \\
\hline $10^{\mathrm{h}} 20^{\prime}$ & $\begin{array}{c}15,1 \\
.\end{array}$ & 23,4 & $\begin{array}{l}\text { Vollstindige Er- } \\
\text { müdung n. 170 Se- } \\
\text { kunden. Erholung }\end{array}$ & 1 h $50^{\prime}$ & 12,2 & 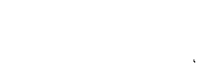 \\
\hline $10^{\mathrm{h}} 35^{\prime}$ & 10,8 & 20,5 & nacn $5-10$ sek. & 2 h $05^{\prime}$ & 11,2 & $\begin{array}{l}\text { Vollständige Er- } \\
\text { müdung nach } 7 \text { Se- } \\
\text { kunden. Erholung } \\
\text { sofort. }\end{array}$ \\
\hline $10^{\mathrm{h}} 50^{\prime}$ & 9,5 & 17 & & $2^{\text {h } 20^{\prime}}$ & 10,6 & \\
\hline $11^{\mathrm{h}} 05^{\prime}$ & 8 & 15,9 & & $2^{\text {h }} 35^{\prime}$ & 10,3 & $\begin{array}{l}\text { Vollständige Er- } \\
\text { müdung nach } 5 \text { Se- } \\
\text { kunden. Erholung } \\
\text { sofort. }\end{array}$ \\
\hline $11^{\mathrm{h}} 20^{\prime}$ & 7 & 14,9 & $\begin{array}{l}\text { Vollständige Er- } \\
\text { müdung n. } 25 \text { Se- } \\
\text { kunden. Erholung } \\
\text { nach } 5 \text { Sekunden. }\end{array}$ & $2^{\text {h }} 50^{\prime}$ & 10,2 & 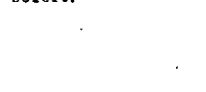 \\
\hline $11^{\mathrm{h}} 35^{\prime}$ & 一 & 13,9 & & $3^{\text {h }} 05^{\prime}$ & 9,7 & $\begin{array}{l}\text { Vollständige Er- } \\
\text { müdung nach } 5 \text { Se- } \\
\text { kunden. Erholung } \\
\text { sofort. }\end{array}$ \\
\hline $11^{\mathrm{h}} 50^{\prime}$ & - & 13,1 & $\begin{array}{l}\text { Vollstaindige Er- } \\
\text { müdung n. } 20 \text { Se- } \\
\text { kunden. Erholung } \\
\text { nach } 5 \text { Sekunden. }\end{array}$ & $3^{\text {h }} 20^{\prime}$ & 8,6 & \\
\hline $12^{\mathrm{h}} 05^{\prime}$ & 一 & 13,1 & 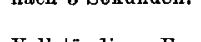 & 3 h $35^{\prime}$ & 5,9 & \\
\hline $12^{\mathrm{h}} 20^{\prime}$ & 一 & 12,9 & $\begin{array}{l}\text { Vollständige } \\
\text { mü- } \\
\text { kundung n. } 15 \text { Se- } \\
\text { n. } 2-5 \text { Sekuolung } \\
\text { Srholunden. }\end{array}$ & $3 h 50^{\prime}$ & $2^{\prime}$ & . \\
\hline $12^{\mathrm{h}} 35^{\prime}$ & $\cdots$ & 12,5 & & $4^{\text {h }} 05^{\prime}$ & - & . \\
\hline $12^{\text {h } 50^{\prime}}$ & - & 12,7 & & - & 一 & \\
\hline $1^{\text {h }}, 05^{\prime}$ & - & 12,4 & $\begin{array}{l}\text { Vollständige Er- } \\
\text { müdung n. } 8 \text { Se- } \\
\text { kunden. Erholung } \\
\text { fast mom } \ominus \text { tan. }\end{array}$ & 一 & - & 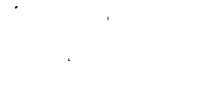 \\
\hline $1^{\text {h }} 20^{\prime}$ & 一 & 12,5 & & - & - & \\
\hline
\end{tabular}


Eine Reaktion der gesamten Muskulatur lässt sich $1^{1 / 2}$ Stunden lang nachweisen; die Minimalreaktion ist nach 6 Stunden erloschen. Am empfindlichsten für den Minimalreiz war bis 11 Uhr 35 Minuten die Schwanzflosse, dann bis 1 Uhr 50 Minuten die Rückenflosse. Bei diesem Karpfen wurden wiederum in Abständen von 30 Minuten (mit einzelnen Ausnahmen) Ermüdungstetanisierungen vorgenommen, die ein ähnliches Bild lieferten, wie es schon bei Tinca beobachtet worden ist. Im Anfang musste 170 Sekunden lang gereizt werden, ehe eine völlige Ermüdung eintrat; die Erholung erfolgte jedoch bedeutend schneller als bei dem Schlei, nämlich bereits nach 5-10 Sekunden. Die zweite Reizung bedurfte schon erheblich kutrzerer Zeit, um die

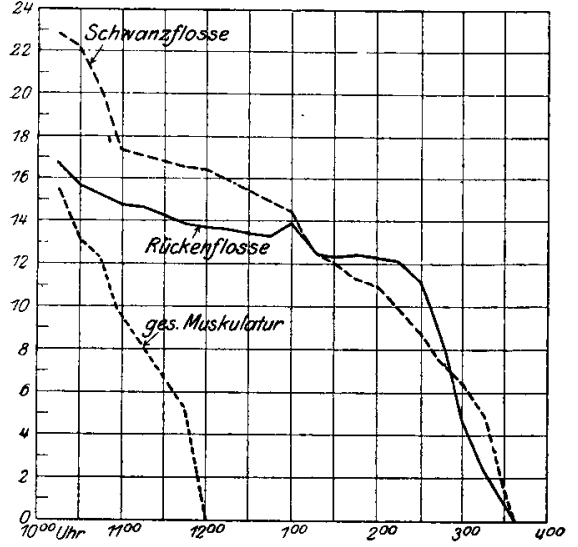

Abb. 3. Versuch am 20. April 1914.

Ermüdung herbeizuführen ( $25 \mathrm{Se}-$ kunden); danach jedoch findet eine gleichmässiger abnehmende Zeitdauer für die Ermüdung Platz. Die Erholungszeit, welche nach jeder Ermudungsreizung notwendig ist, verkürzt sich mit. den kürzeren Reizzeiten. Der Einfluss der Ermüdungsversuche scheint sich, vergleicht man das Verhaiten des ersten Karpfens mit dem des zweiten, nur auf eine etwas stärkere Ausprägung und längere Dauer des ersten Kurvenabfalles zu erstrecken.

Bezüglich des Kurvenverlaufes finden sich die gleichen drei charakteristischen Abstürze zu Beginn des Versuches, in der Mitte und am Ende. Die Kurve nähert sich insofern mehr der des Schleis, als der dritte Absturz ebenfalls am Ende sich vorfindet.

Der Versuch mit dem zweiten Karpfen zeigt obige Kurve (Abb. 3). Versuch am 20. April 1914. Temperatur $21-23^{\circ} \mathrm{C}$. Tötung des Fisches $10 \mathrm{Uhr}$ vormittags.

Da bei dem ersten Karpfen zu Anfang des Versuches eine Reaktion bei äusserstem Rollenabstand an der Schwanzflosse, späterhin an der Rückenflosse und dann schliesslich an beiden zugleich abgelesen worden war, so wurden bei diesem zweiten Karpfen die Ablesungen getrennt von $\Lambda$ nfang an vorgenommen und als Kurven re- 
Versuche über die Dauer der postmortalen Erregbarkeit der Muskulatur usw. 413

gistriert. Hier reagierte zuerst bei äusserstem Rollenabstand die Schwanzflosse; erst nach $3^{1 / 4}$ Stunden trat für kurze Zeit $\left(1^{3 / 4}\right.$ Stunden) die Rückenflosse an ihre Stelle. Die Reaktion hörte bei beiden $\mathrm{zu}$ gleicher Zeit auf.

Der Wechsel der Erregbarkeit zwischen den beiden Flossen zeigt sich bei den zwei untersuchten Fischen in gleicher Weise.

Die gesamte Muskulatur reagierte auf die Reizung mit dem Induktionsstrom noch bis $1 \frac{3}{4}$ Stunden nach dein Tode; die letzte Reaktion erlosch nach $5^{1 / 2}$ Stunden. Die Reaktionsfähigkeit war also bei beiden Karpfen ungefähr gleich lang.

\section{Abramis brama, der Blei.}

Aus der Familie der Cypriniden kam nun noch der Blei (Abramis brama) in zwei Exemplaren zur Untersuchung. Mit dem ersten wurde der Versuch am 6. März, mit dem zweiten am 7. März 1914 vorgenommen. Die Länge beider Versuchsfische war nicht ganz gleich $(23,7$ und $27 \mathrm{~cm})$, die Zimmertemperatur die gleiche $\left(19^{\circ} \mathrm{C}\right.$.) Ein Unterschied bestand insofern zwischen beiden Fischen, als der erste bereits trotz der verhältnismässig kurzen Zeit der Gefangenschaft (5 Tage) von Saprolegnia befallen war, während der zweite körperlich intakt war. Darauf mag auch die verkürate Reaktionszeit beim ersten Blei ( $1 / 2$ Stunde) gegenüber derjenigen beim zweiten, die immerhin auch nur sehr kurz (13/4 Stunden) war, zurückzuführen sein. Besonders auffallend ist das Wiederansteigen der Erregbarkeit beim Versuch am 7. März vom Rollenabstand 9,8 auf 11,2, worauf die Erregbarkeit innerhalb der nächsten Viertelstunde erlosch. Fine Reaktion der gesamten Muskulatur nach dem Tode war überhaupt nicht zu erzielen. Die Kurven fallen ganz aus dem Rahmen derjenigen, welche bei den übrigen Cypriniden erhalten worden sind. Es sollten gerade mit dem Blei noch weitere Versuche vorgenommen werden; jedoch wurden diese durch den Ausbruch des Krieges verhindert. Die Notierungen über die beiden Versuche folgen (Tab. 4 und 5, S. 414).

\section{Anguilla vulgaris, der Aal.}

Zwei Aale wurden untersucht, der erste mit einer Länge von $30 \mathrm{~cm}$ am 6 . Februar 1914 bei einer Zimmertemperatur von $18-20^{\circ} \mathrm{C}$., der zweite mit einer Länge von $48 \mathrm{~cm}$ am 6. Mai 1914 bei einer 
Versuch am 6. März 1914. Temperatur $19^{\circ} \mathrm{C}$. Tötung des Fisches 11 Uhr vormittags.

Tabelle 4.

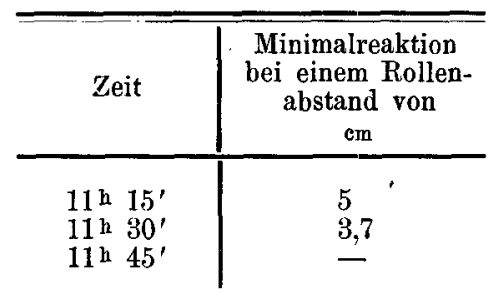

Versuch am 7. März 1914. Temperatur $19^{\circ} \mathrm{C}$. Tötung des Fisches 2 Uhr 45 Min. nachmittags.

\begin{tabular}{|c|c|}
\hline Zeit & $\begin{array}{l}\text { Minimalreaktion } \\
\text { bei einem Rollen- } \\
\text { abstand von } \\
\mathrm{cm}\end{array}$ \\
\hline $\begin{array}{ll}3 \text { h } & 00^{\prime} \\
3 \text { h } & 15^{\prime} \\
3 \text { h } & 30^{\prime} \\
3 \text { h } & 45^{\prime} \\
\text { 4h } & 00^{\prime} \\
\text { 4h } & 15^{\prime} \\
4 \text { h } & 30^{\prime} \\
4 \text { h } & 45^{\prime}\end{array}$ & $\begin{array}{r}15,7 \\
14,7 \\
9,8 \\
10,1 \\
10,6 \\
10,6 \\
11,2 \\
-\end{array}$ \\
\hline
\end{tabular}

Temperatur von $20-21^{\circ} \mathrm{C}$. Die Notierungen uber den Versuch im Februar folgen in Tabelle 6 :

Versuch am 6. Februar 1914. Temperatur $18-20^{\circ}$ C. Tötung des Fisches 10 Uhr vorm.

Tabelle 6 .

\begin{tabular}{|c|c|c|c|c|c|c|}
\hline Zeit & $\begin{array}{c}\text { Reaktion } \\
\text { d. gesamt: } \\
\text { Musku- } \\
\text { latur bei } \\
\text { einem } \\
\text { Rollen- } \\
\text { abstand } \\
\text { von } \\
\text { cm }\end{array}$ & $\begin{array}{c}\text { Minimal- } \\
\text { reaktion } \\
\text { bei einem } \\
\text { Rollen- } \\
\text { abstand } \\
\text { von } \\
\text { cm }\end{array}$ & $\begin{array}{l}\text { Ermüdungs- } \\
\text { versuche }\end{array}$ & Zeit & $\begin{array}{l}\text { Minimal- } \\
\text { reaktion } \\
\text { bei einem } \\
\text { Rollen- } \\
\text { abstand } \\
\text { von } \\
\text { cm }\end{array}$ & $\begin{array}{c}\text { Ermüdungs- } \\
\text { versuche }\end{array}$ \\
\hline $10^{\mathrm{h}} 15^{\prime}$ & 14,9 & 29,6 & & $12 \mathrm{~h} 45^{\prime}$ & 12,2 & \\
\hline $10^{\mathrm{h}} 30^{\prime}$ & 15,2 & 30,2 & $\begin{array}{l}\text { Vollständige Er- } \\
\text { müdung n. } 52 \mathrm{Se-} \\
\text { kunden. Erholung } \\
\text { nach } 7 \text { Sekunden. }\end{array}$ & $1^{\mathrm{h}} 00^{\prime}$ & 12,1 & $\begin{array}{l}\text { Vollständige Er- } \\
\text { müdung nach } 6 \text { bis } \\
7 \text { Sekunden. Erho- } \\
\text { lung n. SSekunden. }\end{array}$ \\
\hline $10^{\mathrm{h}} 45^{\prime}$ & 14,2 & 22,8 & & $1^{\mathrm{h}} 15^{\prime}$ & 11,9 & \\
\hline $11^{\mathrm{h}} 00^{\prime}$ & 13,8 & 21 & $\begin{array}{l}\text { Vollständige Er- } \\
\text { müdung n. } 39 \text { Se- } \\
\text { kunden, Erholung } \\
\text { n. } 7-8 \text { Sekunden. }\end{array}$ & $1^{\text {h }} 30^{\prime}$ & 11,6 & 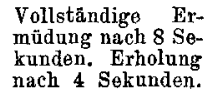 \\
\hline $11^{\mathrm{h}} 15^{\prime}$ & 11,7 & 17,4 & & $1 \mathrm{~h} 45^{\prime}$ & 11,1 & \\
\hline $11^{\mathrm{h}} 30^{\prime}$ & 7,5 & 15,8 & $\begin{array}{l}\text { Vollständige Er- } \\
\text { müdung n.-20 Se- } \\
\text { kunden. Erholung } \\
\text { nach } 19 \text { Sekunden. }\end{array}$ & $2 \mathrm{~h} 00^{\prime}$ & 10,8 & $\begin{array}{l}\text { Vollständige Er- } \\
\text { müdung nach } 4 \text { Se- } \\
\text { kunden. Erholung } \\
\text { nach } 10 \text { Sekunden. }\end{array}$ \\
\hline $11^{\mathrm{h}} 45^{\prime}$ & - & 12 & & $2^{h} 15^{\prime}$ & 6,3 & \\
\hline $12^{\text {h }} 00^{\prime}$ & - & 12,3 & $\begin{array}{l}\text { Vollständige Er- } \\
\text { müdung n. } 12 \text { Se- } \\
\text { kund } \rightarrow \text { n. Erholung } \\
\text { nach } 5 \text { Seknnden. }\end{array}$ & $2 \mathrm{~h} 30^{\prime}$ & - & \\
\hline $12^{\text {h }} 15^{\prime}$ & - & 12,3 & & & - & \\
\hline $12 \mathrm{~h} 30^{\prime}$ & - & 12,3 & $\begin{array}{l}\text { Vollständige Er- } \\
\text { müdung nich 4 So- } \\
\text { kunden. Erholung } \\
\text { n. 4-5 Sekunden. }\end{array}$ & & - & . \\
\hline
\end{tabular}


Versuche über die Dauer der postmortalen Erregbarkeit der Muskulatur usw. $\quad \mathbf{4 1 5}$

Die gesamte Muskulatur reagierte bis $1 \frac{1}{1 / 2}$ Stunden nach Tötung des Fisches; eine Reaktion war überhaupt noch nachzuweisen nach $4^{1 / 4}$ Stunden. Die Reaktion bei äusserstem Rollenabstand wurde an den Brustflossen abgelesen, da diese, nicht die Schwanzflosse wie bei den Cypriniden, die Minimalreaktion zeigten. Der Charakter der Kurve ist ein etwas anderer als der bei den meisten Cypriniden haltene, indem die beiden ersten Kurvenabstürze, der Anfangsand der mittlere Absturz, dicht aneinandergerückt sind, ja fast ineinander überzugehen scheinen. Die Kurve des zweiten Aales zeigt aber wohl, dass man beide trennen kann und auch hier mit Recht drei Abstürze unterscheidet. Hier geht auch der Einfluss der Ermüdungsversuche auf die Ausbildung der stark fallenden Teile der Kurve deutlich hervor, wenn wir das Verhalten beider Aale vergleichen. Dort, wo die Kurve an und für sich keine stark fallende Tendenz zeigt, ist ein Einfluss der Ermüdungsversuche nicht zu erkennen; dort aber, wo ein Absturz zu erwarten ist, ist er um ein Bedeutendes verstärkt.

Um auf die Ermüdungs-

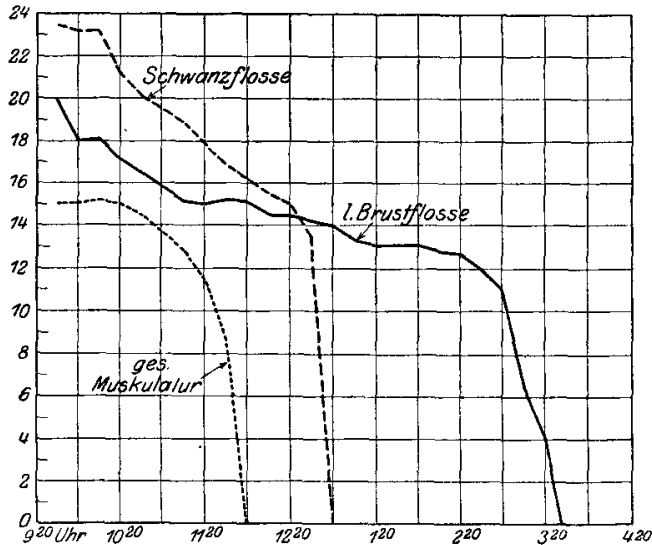

Abb. 4. Versuch am 6. Mai 1914. versuche selbst einzugehen, so zeigt sich hier, dass mit der längeren Zeit, die nach dem Tode verflossen ist, auch die Reizzeit, die zur völligen Ermüdung führt, im allgemeinen kürzer geworden ist; gegen das Ende des Versuches hin wird sie jedoch wieder etwas länger (von 4 Sekunden auf 8 Sekunden); die Erholungzeiten scheinen ganz unabhängig von der Reizdauer zu sein.

Bei dem zweiten Aal dauerte die Reaktionsfähigkeit der gesamten Muskulatur $2^{3} / 4$ Stunden; die letzte Reaktion konnte nach 6 Stunden abgelesen werden. Wie schon gesagt, ist hier der zweite Absturz als von dem ersten getrennt zu erkennen, wenn auch nur durch die Dauer einer Viertelstunde. Da beim zweiten Aal die Schwanzflosse anfangs die Reaktion bei äusserstem Rollenabstand zeigte, so wurden Notierungen 
der Reaktion der Brustflossen gesondert vorgenommen und als Kurve aufgezeichnet.

\section{Esox lucius, der Hecht.}

Vom Hecht wurde nur ein Exemplar untersucht, und zwar am 27. Februar 1914. Die Länge desselben betrug $47,5 \mathrm{~cm}$. Bei diesem Hecht wurden auch gleichzeitig Ermüdungsversuche vorgenommen.

Versuch am 27. Februar 1914. Temperatur $17,5-18,5^{\circ} \mathrm{C}$. Tötung des Fisches 10 Uhr vormittags.

Tabelle 7 .

\begin{tabular}{|c|c|c|c|c|c|c|}
\hline Zeit & $\begin{array}{l}\text { Reaktion } \\
\text { d. gossamt. } \\
\text { Musku- } \\
\text { litur bei } \\
\text { einem } \\
\text { Rollen- } \\
\text { abstand } \\
\text { von } \\
\text { cm }\end{array}$ & $\begin{array}{l}\text { Minimal- } \\
\text { reaktion } \\
\text { bei einem } \\
\text { Rollen- } \\
\text { ibbstand } \\
\text { von } \\
\quad \text { cm }\end{array}$ & $\begin{array}{l}\text { Ermuldungs- } \\
\text { versuche }\end{array}$ & Zeit & $\begin{array}{l}\text { Minimal- } \\
\text { re:thtion } \\
\text { bei Ainein } \\
\text { Rollen- } \\
\text { abstilud } \\
\text { von } \\
\text { em }\end{array}$ & $\begin{array}{l}\text { Ermüdungrs- } \\
\text { versuche }\end{array}$ \\
\hline $10^{\mathrm{h}} 15^{\prime}$ & 16 & 25,4 & & 12 h $00^{\prime}$ & 14 & $\begin{array}{l}\text { Vollitïndige Er- } \\
\text { müdung nach 14 Se- } \\
\text { kundent. Erholunh } \\
\text { n. 10-12 Sekunden. }\end{array}$ \\
\hline $10^{\text {h }} 30^{\prime}$ & 14,3 & 24,8 & $\begin{array}{l}\text { Vollstindige Er- } \\
\text { müdung n. 127 se- } \\
\text { kunden. Erholung } \\
\text { nach 10 Sekunden }\end{array}$ & $12 \mathrm{~h} 15^{\prime}$ & 13,3 & \\
\hline $10^{\mathrm{h}} 45^{\prime}$ & - & 19,3 & & $12^{\mathrm{h}} 30^{\prime}$ & 12,6 & $\begin{array}{l}\text { Ermüdung wegen } \\
\text { sehr geringer Re- } \\
\text { aktion nicht mehr } \\
\text { feststellbar. }\end{array}$ \\
\hline $11^{\mathrm{h}} \quad 00^{\prime}$ & - & 18 & $\begin{array}{l}\text { Vollständige Fr- } \\
\text { middung n. } 5.5 \text { Se- } \\
\text { kunden. Erhulung } \\
\text { nach } 15 \text { Sekunden. }\end{array}$ & $12^{\text {h }} 45^{\prime}$ & 4,5 & \\
\hline $11^{\text {h }} 15^{\prime}$ & - & 16,3 & & $1^{\text {h }} 00^{\prime}$ & - & \\
\hline $11^{\mathrm{h}} 30^{\prime}$ & 一 & 15,9 & $\begin{array}{l}\text { Vollständige Er- } \\
\text { nuüdung n. } 37 \text { sie- } \\
\text { kunden. Erholung } \\
\text { nach } 15-20 \text { Sek. }\end{array}$ & - & - & \\
\hline $11^{\text {h }} 45^{\prime}$ & - & 14,2 & & - & - & \\
\hline
\end{tabular}

Die gesamte Muskulatur reagierte also noch nach $1 / 2$ Stunde; die letzte Reaktion wurde nach $2^{3 / 4}$ Stunden verzeichnet. Aus der Tabelle geht hervor, dass auch hier drei stärkere Abfälle zu beobachten sind: am Anfang, in der Mitte und am Schluss der Versuchszeit.

Bei den Ermüdungsversuchen ist eine verhältnismässig gleichmässige Verkürzung der zur Frmüdung notwendigen Reizzeit zu beobachten. Auffällig ist die im Vergleich zu den übrigen Fischen besonders zu Beginn verhältnismässig lange Erholungszeit, die bis zum Wiederauftreten einer Reaktion notwendig ist.

Perca fluviatilis, der Barsch.

Es wurde nur ein Barsch untersucht am 4. März 1914 bei einer Zimmertemperatur von $18,5-19,5^{\circ} \mathrm{C}$. Das Tier hatte eine 
Versuche über die Dauer der postmortalen Erregbarkeit der Muskulatur usw. 417

Länge von $25,5 \mathrm{~cm}$. Die Zeitdauer der Reaktionsfähigkeit der gesamten Muskulatur betrug weniger als 15 Minuten; es reagierten zuerst nur sämtliche Flossenmuskeln. Die Reaktion bei äusserstem Rollenabstand zeigten die Brustflossen; diese konnte während einer Zeit von $1 \frac{1 / 4}{4}$ Stunden abgelesen werden. Der einmalige Ermüdungsversuch ergab, dass nach 38 Sekunden langer Reizung eine völlige Ermüdung herbejgeführt werden konnte, dass die Erholungszeit 5 Sekunden betrug.

Versuch am 4. März 1914. Tötung des Fisches 10 Uhr 45 Minuten vormittags.

Tabelle 8 .

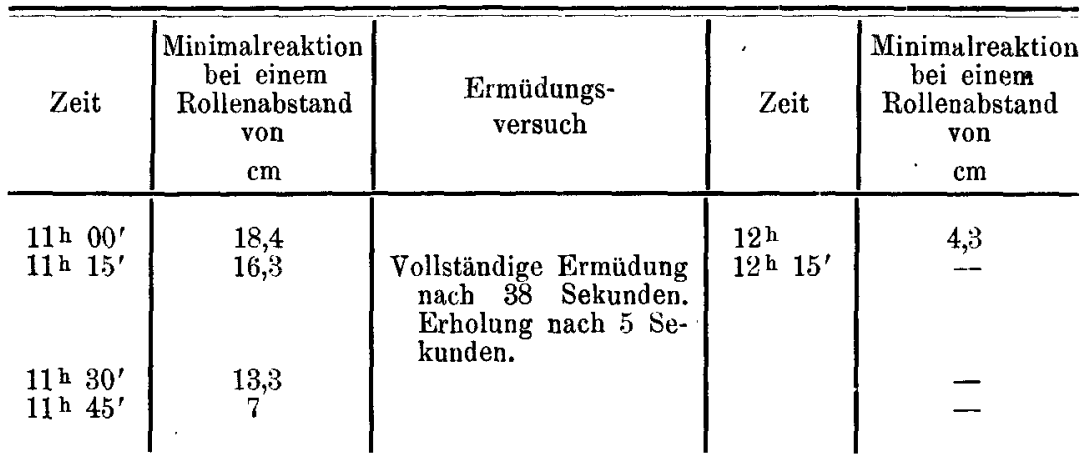

Trutta iridea, die Regenbogenforelle.

Die beiden Versuche mit Regenbogenforellen wurden am 20. Februar und 3. März 1914 vorgenommen. Bei dem ersten Versuch herrschte im Zimmer eine Temperatur von $19-20^{\circ} \mathrm{C}$., bei dem zweiten von $21^{\circ}$ C. Der erste Fisch war $23,3 \mathrm{~cm}$ lang, der zweite $21,5 \mathrm{~cm}$. Bei beiden Forellen war die Dauer der Reaktionen sehr kurz; der Versuch am 20. Februar ergab eine Reaktion der gesamten Muskulatur bereits nach 15 Minuten nicht mehr, eine Reaktion der Flossen konnte bei $10 \mathrm{~cm}$ Rollenabstand noch nach 15 Minuten abgelesen werden; nach 30 Minuten war eine solche schon nicht mehr nachzuweisen. Die zweite Forelle reagierte noch nach 15 Minuten mit ihrer gesamten Muskulatur; die Reaktionsfähigkeit überhaupt erlosch nach 1 Stunde.

Versuch am 20. Februar 1914. Temperatur $19-20^{\circ}$ C. Tötung des Fisches 10 Uhr 15 Minuten vormittags. 


\section{Tabelle 9 .}

\begin{tabular}{c|c|c}
\hline Zeit & $\begin{array}{c}\text { Reaktion der gesamten } \\
\text { Muskulatur bei einem } \\
\text { Rollenabstand von } \\
\mathrm{cm}\end{array}$ & $\begin{array}{c}\text { Minimalreaktion bei } \\
\text { einem Rollenabstand von } \\
\mathrm{cm}\end{array}$ \\
\hline $10 \mathrm{~h} 30^{\prime}$ & - & 10 \\
$10^{\mathrm{h} 45^{\prime}}$ & - & -
\end{tabular}

Versuch am 3. März 1914. Temperatur $21^{\circ} \mathrm{C}$. Tötung des Fisches 4 Uhr 45 Minuten nachmittags.

Tabelle 10.

\begin{tabular}{c|c|c}
\hline Zeit & $\begin{array}{c}\text { Reaktion der gesamten } \\
\text { Muskulatur bei einem } \\
\text { Rollenabstand von } \\
\text { em }\end{array}$ & $\begin{array}{c}\text { Minimalreaktion bei } \\
\text { einem Rollenabstand von } \\
\text { om }\end{array}$ \\
\hline 5h $10^{\prime}$ & 13 & 23,8 \\
5 h $25^{\prime}$ & - & 15 \\
5 h $40^{\prime}$ & - & 7,8 \\
5 h $55^{\prime}$ & - & -
\end{tabular}

An den Kurven sind Besonderheiten nicht zu erkennen; sie fallen bei beiden Forellen gleichmässig steil ab.

\section{Trutta fario, die Bachforelle.}

Ein Unterschied zwischen der Regenbogenforelle und der Bachforelle in der Dauer der Reaktionsfähigkeit der Muskulatur besteht nicht. Bei dem unter gleichen Bedingungen (Länge $22 \mathrm{~cm}$ ) wie bei der zweiten Regenbogenforelle am 3. März 1914 vorgenommenen Versuch ergab sich für die gesamte Muskulatur eine Reaktionsfähigkeit von $1 / 4$ Stunde. Die letzte Reaktion wurde nach $1 / 2$ Stunde nachgewiesen. Die Form der erhaltenen Kurve entspricht somit den bei den Regenbogenforellen gewonnenen.

Bei der zweiten Regenbogenforelle und der Bachforelle war sofort nach dem Tode eine blitzartige Zuckung des ganzen Körpers auf den Reiz mit dem Induktionsstrom zu konstatieren.

Versuch am 3. März 1914. Temperatur $21^{\circ} \mathrm{C}$. Tötung des Fisches 4 Uhr nachmittags. 
Versuche über die Dauer der postmortalen Erregbarkeit der Muskulatur usw. 419

Tabelle 11 .

\begin{tabular}{c|c|c}
\hline Zeit & $\begin{array}{c}\text { Reaktion der gesamten } \\
\text { Muskulatur bei einem } \\
\text { Rollenabstand von } \\
\text { em }\end{array}$ & $\begin{array}{c}\text { Minimalreaktion bei } \\
\text { einem Rollenabstand von } \\
\mathrm{cm}\end{array}$ \\
\hline $\begin{array}{c}4 \text { h } 15^{\prime} \\
4 \text { h } 30^{\prime}\end{array}$ & 14 & 21,6 \\
4 h $45^{\prime}$ & - & -
\end{tabular}

\section{Zusammenfassung.}

Bei vergleichender Betrachtung der vorstehend angeführten Versuche sei zuerst die verschiedene Zeitdauer der Reaktionsfähigkeit bei den einzelnen Formen zusammengestellt in der Reihenfolge der Länge dieser Zeiten:

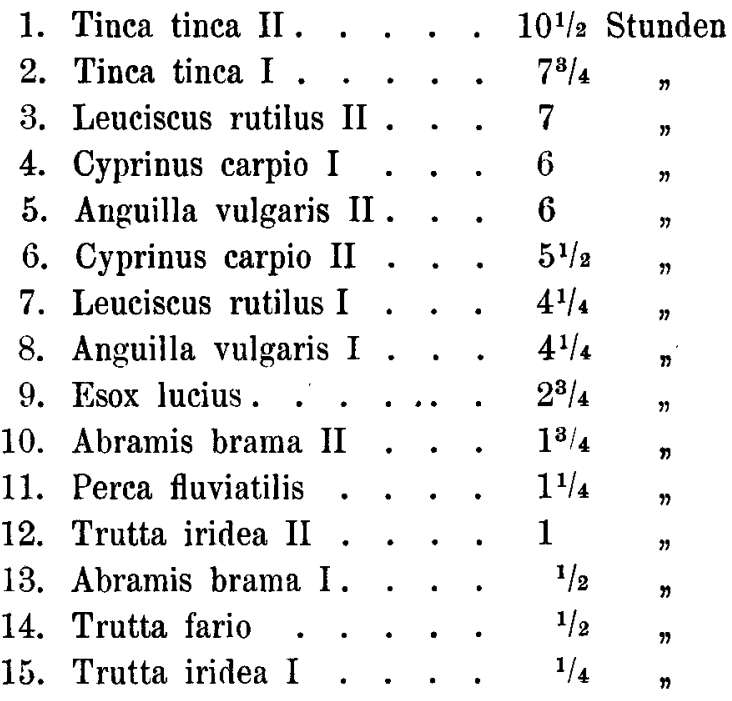

Wir können demnach zwei Gruppen bilden, in deren eine Fische mit längerer Reaktionsdauer gehören $(i-8)$, in deren andere diejenigen mit kürzerer Reaktionsfähigkeit fallen $(9-15)$. Im wesentlichen gehören zur ersten Gruppe Angehörige der Familie der Cypriniden; dazu tritt der Aal. Zur zweiten Gruppe gehören vor allem die Salmoniden, Hecht, Barsch, und als einziger Vertreter der Cypriniden der Blei ${ }^{1}$ ). Es ist interessant, dass die erste Gruppe die so-

1) Über den Blei müssen wohl noch weitere Versuche entscheiden. 
genannten Friedfische enthält, während in der zweiten sich die Raubfische vorfinden.

Im Hinblick auf die eingangs dieser Arbeit dargelegten Erwägungen wollen wir die in meinen Versuchen gefundenen Mittelwerte der Reaktionsdauer nach dem Tode mit der Intensität des Stoffwechsels vergleichen, welche Lindstedt und Knauthe durch Respirationsversuche festgestellt haben. Wir berechnen zu diesem Behufe für die betreffenden Fischarten aus Tab. IV, Stab 14 von Lindstedt's Abhandlung (Zeitschrift f. Fischerei Bd. 14 S. 245) die Zeit, innerhalb derer der betreffende Fisch $100 \mathrm{~g}$ Kalorien auf 1 qdem Körperoberfläche bei $15^{\circ} \mathrm{C}$. verbraucht, und vergleichen diese Zeit mit der Reaktionsdauer im Mittel meiner Versuche.

\begin{tabular}{|c|c|c|}
\hline Fischart & $\begin{array}{l}\text { Reaktions- } \\
\text { dauer }\end{array}$ & $\begin{array}{l}\text { Zeit des Verbrauchs } \\
\text { von } 100 \mathrm{~g} \text { Kalorien } \\
\text { in Stunden }\end{array}$ \\
\hline Tinca tinca & $\emptyset, 12$ & 8,23 \\
\hline Cyprinus carpio . & 5,75 & 3,79 \\
\hline Esox lucius . . & 2,75 & 5,62 \\
\hline Perca fluviatilis. & 1,25 & 7,06 \\
\hline Trutta iridea . & 0,63 & 1,67 \\
\hline
\end{tabular}

Im allgemeinen weisen diese Zablen auf eine nahe Beziehung der Intensität des Stoffwechsels zur Dauer des Überlebens der Muskeln; nur bei Hecht und Barsch weichen die Werte stärker ab.

Weitere Unterschiede zwischen den einzelnen Arten ergeben sich aus der Form der Kurven. Wir hatten gesehen, dass bei einem Teil der untersuchten Fische die Kurven allmählich abfielen, dass dieser langsame Abfall jedoch durch drei Abstürze oder doch stärkere Abfälle unterbrochen wurde, während diese drei Abstürze bei dem anderen Teil nicht beobachtet werden konnten. Diesen ersten Typus der Kurvenform zeigten folgende Fische: Tinca tinca, Leuciscus rutilus, Cyprinus carpio und auch wohl Anguilla vulgaris. Da man auch bei der kürzer und steiler verlaufenden Kurve von Esox lucius ebenfalls drei Abstürze unterscheiden kann, so dürfen wir auch wohl den Hecht zu dieser Gruppe rechnen. Vielleicht zeigt gerade dieses Beispiel, dass bei genauerer Analyse der einzelnen Kurven der übrigeu Fische bzw. bei weiterem Auseinanderziehen derselben durch andere Versuchsanordnung (zum Beispiel häufigeres Ablesen der Reaktion in kürzeren Zwischenräumen) auch diese vielleicht den gleichen mehrstufigen Charakter 
besitzen würden wie die von den meisten Cypriniden erhaltenen Kurven. $\mathrm{Ob}$ also diese Unterscheidung zwischen den Arten, deren Reizbarkeit mit wechselnder Gesch windigkeit abfällt, und denen, die diesen Charakter nicht zeigen, berechtigt ist, lässt sich endgültig noch nicht entscheiden.

Vergleicht man, wie sich der Rollenabstand bei der schwächsten Reaktion zu Beginn der einzelnen Versuche verhält, so könnte man annehmen, dass dies abhängig wäre von der Dauer der Reizbarkeit resp. der Länge der Kurve. Dem ist aber nicht ganz so. Wir erhalten nämlich folgende Reihe ${ }^{1}$ ):

1. Tinea tinca II . . . . . . . . $30,5 \mathrm{~cm}$

2. Anguilla vulgaris I . . . . . . 29,6 "

8. Leuciscus rutilus II . . . . . . 29,5

4. Esox lucius . . . . . . . . . 25,4,

5. Cyprinus carpio I . . . . . . . 24,1,

6. Trutta iridea II . . . . . . . . 23,8"

7. Anguilla vulgaris II . . . . . . 23,5

8. Cyprinus carpio II . . . . . . . 22,8 "

9. Trutta fario . . . . . . . . 21,6 ,

10. Leuciscus rutilus I . . . . . . 21,2,

11. Perca fluviatilis . . . . . . . 18,4"

12. Abramis brama II . . . . . . 15,7,

13. Trutta iridea I . . . . . , 10 ,

14. Abramis brama I . . . . . . 5 "

Es ist anzunebmen, dass die anfangs zur Auslösung der Reaktion erforderliche Stromstärke von der zufällig wechselnden Entfernung des Elektrodendrahtes vom motorischen Nerven der Flossenmuskeln abhängt, also keine prinzipielle Bedeutung hat. Jedenfalls ist der Verlauf der Kurve von der Anfangsweite des Rollenabstandes im wesentlichen unabhängig.

1) Tinca tinca I kann nicht zum Vergleich herangezogen werden. 